\title{
Diversidad y distribución de los crustáceos estomatópodos y decápodos en el estuario del río Gallegos (Santa Cruz, Argentina)
}

\author{
Eduardo R. Torres \& Fabiana A. Vargas \\ Universidad Nacional de la Patagonia Austral (UNPA-UARG). Lisandro de la Torre 1070. (9400) Río Gallegos. Santa Cruz. \\ Argentina; edtorres@criba.edu.ar
}

Recibido 11-X-2005. C Corregido 08-XI-2006. Aceptado 16-III-2007.

\begin{abstract}
Diversity and distribution of stomatopod and decapod crustaceans in the estuary of Gallegos river (Santa Cruz-Argentina). We studied the diversity and distribution of stomatopod and decapod crustaceans from the estuary of Gallegos River, Argentina. Decapods were found at all stations, while stomatopods and anomurans were found only at the exterior station. There was a marked difference in diversity, the exterior station being more diverse. A total of six species were found (five decapods and one stomatopod), a low value when compared with the surrounding sea. Abundance was variable at stations, depending on the type of bottom. Rev. Biol. Trop. 55 (Suppl. 1): 113-120. Epub 2007 June, 29.
\end{abstract}

Key words: distribution, diversity, estuary of Gallegos River, Santa Cruz province, Argentina.

El estuario del río Gallegos, Argentina, constituye un área de escasa importancia económica, asentándose únicamente pesquerías artesanales dedicadas a pocas especies de peces como róbalo (Eleginops maclovinus (Valenciennes, 1830)) y pejerreyes (Austroatherina smitti (Lahielle, 1929) y Odontesthes (Austromenidia) nigricans (Richardson, 1848)), además de explotar moluscos bivalvos como mejillones (Mytilus chilensis Hupé, 1854) y cholga (Aulacomya ater (Molina, 1792)) (Caille et al. 1995, Caille 1996, Braccalenti 2000, Ferrari 2000, Pellanda y Fernández 2000, Perroni 2000, Torres et al. 2006). El área es una zona de desove y cría de los recursos mencionados, como también un área de asentamiento de una gran cantidad de aves migratorias que nidifican y se alimentan en el mismo (Ferrari y Albrieu 2000, Torres y Vargas 2005).

Los crustáceos constituyen uno de los principales grupos zoológicos del mar, ya sea como integrantes de las comunidades marinas o como recurso en la economía del hombre.
Además se les encuentra en el contenido estomacal de peces que tienen importancia en las pesquerías, o de aves migratorias de la zona. Por estas razones es importante conocer el número de especies y la distribución de las mismas, además de ubicar el nicho ecológico dentro de la comunidad de vida a la cual pertenecen (Boschi 1964).

En el estuario se desarrollan también varias poblaciones de invertebrados bentónicos que representan una fuente importante de alimento para las especies anteriormente mencionadas. Estos están constituidos principalmente por moluscos, crustáceos y poliquetos (Pittaluga 2003, Torres et al. 2005).

Las observaciones y los datos disponibles sobre los crustáceos decápodos del mar argentino en su mayor parte provienen de investigaciones realizadas en el extranjero, con material obtenido por expediciones oceanográficas, de canjes con museos y de ejemplares recogidos por naturalistas que han recorrido nuestro extenso litoral marítimo (Boschi 1964). Existen pocos trabajos que traten la carcinofauna de la 
Patagonia Sur, y es muy complejo su estudio en estas latitudes donde no se cuenta con información de la biodiversidad existente en ciertos grupos biológicos (Torres 2005).

Las poblaciones de crustáceos bentónicos del estuario tienen un significado fundamental, tanto por su aprovechamiento directo, como por su rol en la alimentación de peces de importancia comercial y otros animales, también por su función como carroñeros (Vinuesa 2005a).

En el presente trabajo se registra la presencia de las especies de crustáceos decápodos y estomatópodos halladas en el estuario del río Gallegos y se discuten datos de su distribución y diversidad.

\section{MATERIALES Y MÉTODOS}

El material estudiado proviene de siete campañas realizadas en cuatro estaciones de verano, ubicadas sobre el margen sur del estuario del río Gallegos (5136’33”' S, 69¹3'36.7”' W) (Santa Cruz, Argentina) (Fig. 1), entre los años 1998-2004.
Se muestreó una estación exterior (Punta Loyola-E4), dos medias (Río Chico-E3 y Río Gallegos-E2) y una interior (Güer AikeE1). Por otra parte se contó con la oportunidad de estudiar la Sección Crustáceos de la Colección de Referencia de la Cátedra de Zoología de la Universidad Nacional de la Patagonia Austral (UNPA-UARG), que incluye material proveniente de campañas realizadas en la zona por otros investigadores, con lo que se completó en gran medida la distribución geográfica de los crustáceos dentro del estuario.

Los muestreos se hicieron por medio de transectos de $2000 \mathrm{~m}^{2}$ paralelos a la línea de costa cubriendo los niveles superior, medio e inferior del intermareal, recolectando el material en forma directa y conservándolo en formaldehído 5 \% diluido en agua de mar. Las especies se determinaron en laboratorio hasta el nivel de especie siguiendo a Boschi et al. (1992) y certificando su presencia en la zona utilizando a Vinuesa (1977), Boschi (1979, 1997, 2000), Retamal (1981, 2000), Spivak (1997) y Romero (2002).

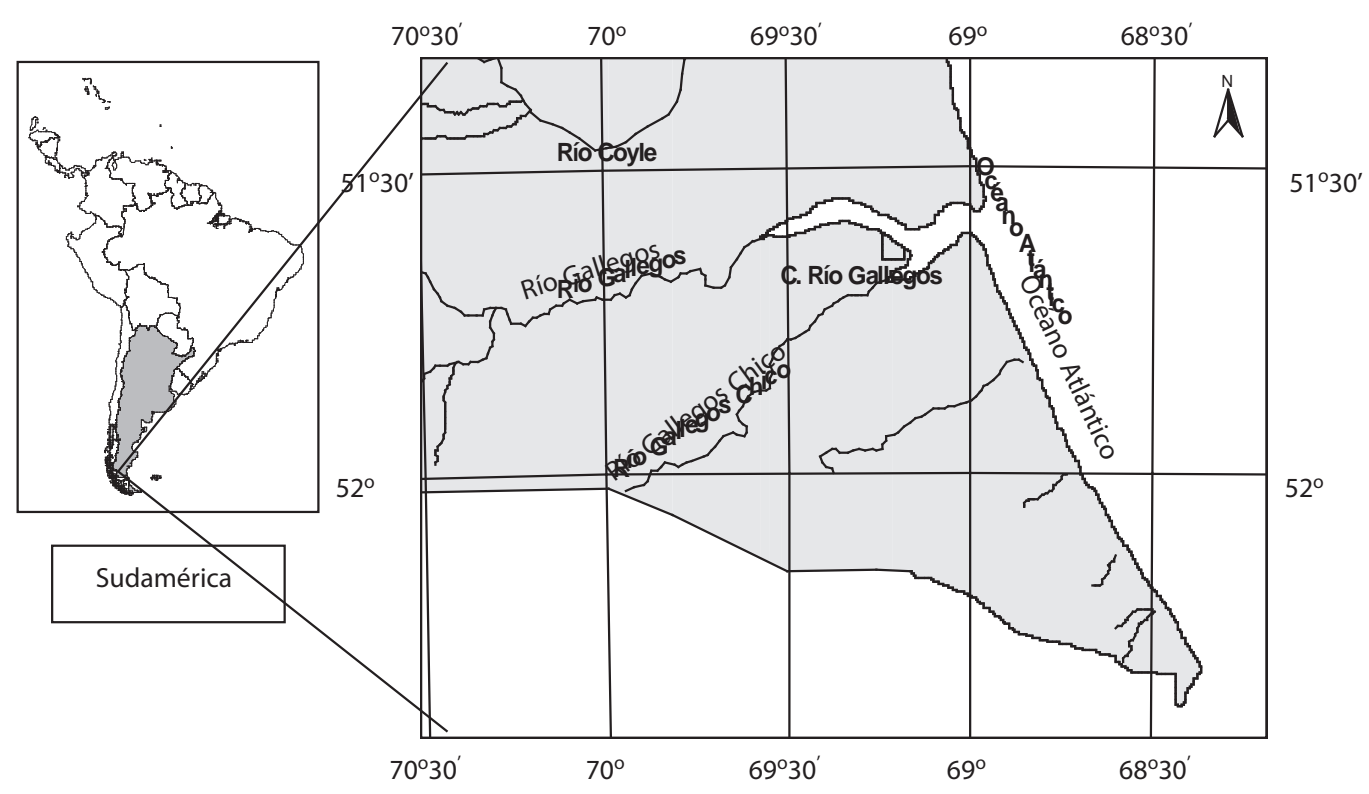

Fig. 1. Ubicación del estuario del río Gallegos (51³6’33”S y 69¹3’36.7’W) en la costa de la provincia de Santa Cruz, Argentina.

Fig. 1. Location of the estuary of Gallegos river (5136'33’'S y 69¹3'36.7’'W) in Santa Cruz province, Argentina. 
Las principales medidas obtenidas, con un calibrador Digimess (DS $=0.05 \mathrm{~mm}$ ) en cada ejemplar, corresponden al largo del caparazón (LC) tomado desde la punta del rostro o región frontal hasta el margen posterior del caparazón, y el ancho del caparazón (AC) representado por la distancia máxima entre los bordes del caparazón (Boschi 1964). Cada ejemplar fue sexado y se tomaron datos del sustrato donde se hallaron.

\section{RESULTADOS}

Se registró un total de seis especies para el estuario del río Gallegos, agrupándose en cinco especies del orden Decapoda repartidas en dos especies del infraorden Anomura (Paralomis granulosa (Jacquinot, 1847) y Lithodes confundens Macpherson, 1988) y tres especies del infraorden Brachyura (Peltarion spinosulum (White, 1843), Eurypodius latreillei Guérin, 1828 y Halicarcinus planatus (Fabricius, 1775)). Además se recolectó una especie del orden Stomatopoda (Heterosquilla
(H.) polydactyla (von Martens, 1881)) (Cuadro 1). Mientras que los Brachyura se presentaron en las cuatro estaciones, los Anomura y los Stomatopoda solo estuvieron presentes en la estación exterior (Punta Loyola-E4). Halicarcinus planatus solo fue hallado en la estación externa (Punta Loyola-E4) y en la estación interna (Güer Aike-E1) donde fue la única especie registrada para esa zona.

Se obtuvieron 178 individuos repartidos entre las seis especies registradas (Cuadro 2). $H$. planatus presentó la mayor frecuencia de presencia, siendo esta $42.1 \%$, mientras que $P$. spinosulum y E. latreillei representaron 20.8 \% y 10.7 \% respectivamente. Los anomuros, P. granulosa y L. confundens presentaron frecuencias similares, siendo ésta $12.9 \%$. El estomatópodo $H$. (H.) polydactyla solo presentó una frecuencia de $0.6 \%$ (Fig. 2).

Las medidas del AC y LC obtenidas no se alejan de los valores observados por Boschi et al. (1992), Boschi (1979, 1997, 2000), Retamal (1981, 2000) y Romero (2002) (Cuadro 2).

Con relación a la abundancia, pudo verse que en la estación interior (Güer Aike-E1) $H$.

CUADRO 1

Especies de crustáceos Decapoda y Stomatopoda recolectadas en las diferentes estaciones de muestreo en el estuario del río Gallegos (Santa Cruz-Argentina).

TABLE 1

Species of Decapoda and Stomatopoda collected at the different stations in the estuary of Gallegos river (Santa Cruz-Argentina).

\begin{tabular}{|c|c|c|c|c|}
\hline & $\begin{array}{l}\text { Güer Aike } \\
\text { (E1) }\end{array}$ & $\begin{array}{c}\text { Río Gallegos } \\
\text { (E2) }\end{array}$ & $\begin{array}{l}\text { Río Chico } \\
\text { (E3) }\end{array}$ & $\begin{array}{c}\text { Punta Loyola } \\
\text { (E4) }\end{array}$ \\
\hline \multicolumn{5}{|l|}{ Orden Stomatopoda } \\
\hline \multicolumn{5}{|l|}{ Familia Lysiosquillidae } \\
\hline Heyerosquilla (H.) polydactyla (von & & & & $\mathrm{X}$ \\
\hline \multicolumn{5}{|l|}{ Martens, 1881) } \\
\hline \multicolumn{5}{|l|}{ Orden Decapoda } \\
\hline \multicolumn{5}{|l|}{ Infraorden Anomura } \\
\hline \multicolumn{5}{|l|}{ Familia Lithodidae } \\
\hline Paralomis granulosa (Jacquinot, 1847) & & & & $\mathrm{X}$ \\
\hline Lithodes confundens Macpherson, 1988 & & & & $\mathrm{X}$ \\
\hline \multicolumn{5}{|l|}{ Infraorden Brachyura } \\
\hline \multicolumn{5}{|l|}{ Familia Majidae } \\
\hline Eurypodius latreillei Guérin, 1828 & & $\mathrm{X}$ & $\mathrm{X}$ & $\mathrm{X}$ \\
\hline \multicolumn{5}{|l|}{ Familia Hymenosomatidae } \\
\hline Halicarcinus planatus (Fabricius (1775) & $\mathrm{X}$ & & & $\mathrm{X}$ \\
\hline \multicolumn{5}{|l|}{ Familia Atelecyclidae } \\
\hline Peltarion spinosulum (White, 1843) & & $\mathrm{X}$ & $\mathrm{X}$ & $\mathrm{X}$ \\
\hline
\end{tabular}


CUADRO 2

Número total de individuos capturados por especie y promedio del AC y LC expresados en milímetros para el estuario del río Gallegos (Santa Cruz-Argentina).

TABLE 2

Total number of individuals collected per species, and their average of the AC and LC expressed in millimeters from the estuary of Gallegos river (Santa Cruz-Argentina).

Heterosquilla (H.) polydactyla
Paralomis granulosa
Lithodes confundens
Eurypodius latreillei
Peltarion spinosulum
Halicarcinus planatus

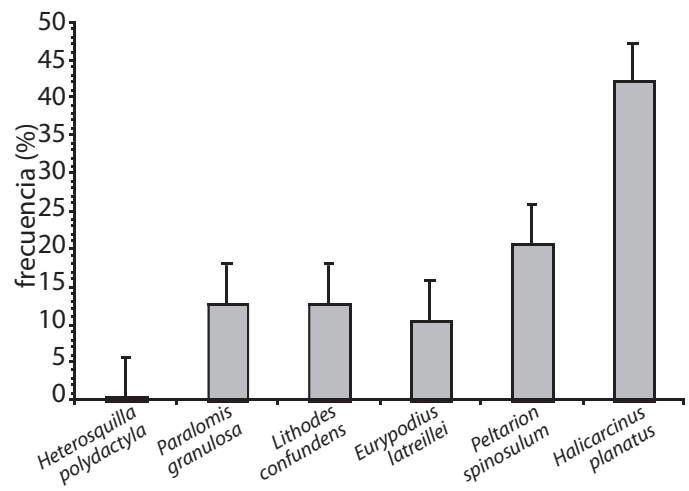

Fig. 2. Frecuencia de presencia de las seis especies de crustáceos decápodos y estomatópodos registrados para el estuario del río Gallegos (Santa Cruz-Argentina).

Fig. 2. Frequency of presence for the six species of decapod and stomatopod crustaceans registered for the estuary of Gallegos river (Santa Cruz-Argentina).

planatus presentó $0.026 \mathrm{ind} / \mathrm{m}^{2}$, mientras que en la estación exterior (Punta Loyola-E4) presentó $0.012 \mathrm{ind} / \mathrm{m}^{2}$. En la estación media (Río Gallegos-E2) E. latreillei y P. spinosulum presentaron $0.003 \mathrm{ind} / \mathrm{m}^{2}$, y en la estación media (Río Chico-E3) estas especies presentaron 0.002 ind/ $\mathrm{m}^{2}$ y $0.014 \mathrm{ind} / \mathrm{m}^{2}$ respectivamente. La estación exterior (Punta Loyola-E4) registró el mayor número de especies, siendo la abundancia de $P$. granulosa y $L$. confundens $0.012 \mathrm{ind} / \mathrm{m}^{2}$, de $P$. spinosulum $0.002 \mathrm{ind} / \mathrm{m}^{2}$, de E. latreillei 0.005 ind $/ \mathrm{m}^{2}$, y de $H$. (H.) polydactyla $0.001 \mathrm{ind} / \mathrm{m}^{2}$.
$\mathrm{AC}(\mathrm{mm})$

$\mathrm{LC}(\mathrm{mm})$

$41.0( \pm 0.05)$

$39.0( \pm 0.05)$

$27.5( \pm 0.05)$

$26.0( \pm 0.05)$

$10.5( \pm 0.05)$

$50.0( \pm 0.05)$

$44.5( \pm 0.05)$

$42.0( \pm 0.05)$

$26.0( \pm 0.05)$

$9.0( \pm 0.05)$

La diversidad registrada fue mayor en la estación exterior (Punta Loyola-E4) donde fueron encontradas la totalidad de las especies, haciéndose menor hacia el interior del estuario donde, en la estación interna (Güer Aike-E1), sólo se registró $H$. planatus. En las estaciones medias (Río Gallegos-E2 y Río Chico-E3) sólo se registró la presencia de E. latreillei y $P$. spinosulum con valores de abundancia disímiles (Fig. 3).

Tanto en E. latreillei como en P. spinosulum se pudo observar que existía una relación de sexos de $1: 1$, mientras que en $H$. planatus todos

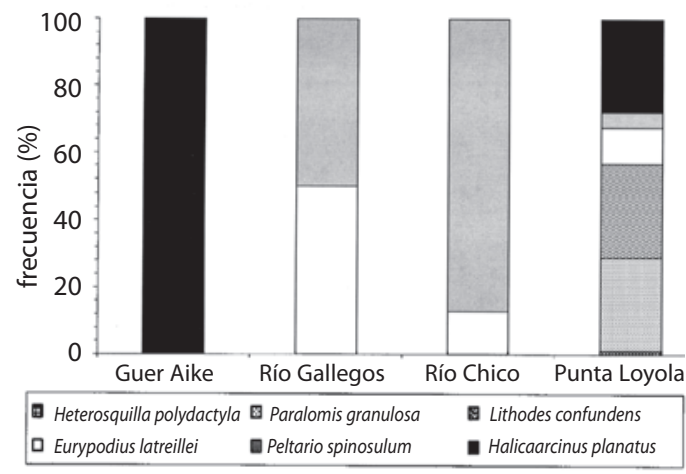

Fig. 3. Frecuencia de presencia de las seis especies registradas en el estuario del río Gallegos (Santa Cruz-Argentina) para cada una de las diferentes estaciones de muestreo analizadas.

Fig. 3. Frequency of presence for the six species registered in the estuary of Gallegos river (Santa Cruz-Argentina) for each of the different sampling stations analyzed. 
los ejemplares recolectados eran hembras. Los anomuros $P$. granulosa y $L$. confundens eran en su totalidad machos. El estomatópodo no pudo ser sexado.

Los tipos de fondos observados en las estaciones fueron muy diversos, pudiéndose apreciar fondo de arenisca consolidada con grandes rocas del mismo material en la estación interior (Güer Aike-E1), fondo de limo arcilla con cantos rodados medios en la estación media (Río Gallegos-E2), fondos de limo arcilla en la estación media (Río Chico-E3) y fondos de cantos rodados medios a grandes y arenas medias a gruesas en la estación exterior (Punta Loyola-E4).

\section{DISCUSIÓN}

El estuario del río Gallegos ha sido incluido en la Provincia Magallánica, con una influencia manifiesta de aguas templado-frías. El reducido número de especies de crustáceos en esta provincia ha sido bien documentado para la costa de Argentina (Boschi 1964, 1979, Vinuesa 1977, Spivak 1997), pero faltando datos específicos para la costa de la provincia de Santa Cruz.

De la investigación surge que el número reducido de especies de crustáceos no es una excepción para la zona estudiada dado que solo fueron registradas seis especies. Ese valor es verdaderamente bajo comparado con la totalidad de crustáceos estomatópodos y decápodos citados para el mar argentino.

Los crustáceos presentaron un asentamiento diferencial en las estaciones analizadas, atribuyendo dicho suceso principalmente a los tipos de fondo, dado que $H$. planatus prefiere ambientes donde se encuentren rocas o canto rodado grande donde pueda resguardarse, con poca o moderada disposición de limo (Vinuesa y Ferrari 2003). E. latreillei es más abundante en zonas con fondos mixtos de canto rodado y limo arcilla, mientras que $P$. spinosulum es más frecuente y abundante en fondos de limo-arcilla. Los anomuros ( $P$. granulosa y $L$. confundens) fueron más frecuentes en fondos de arenas medias a gruesas. Con respecto a $H$. (H.) polydactyla, el tipo de sedimento es condicional para la construcción de sus galerías, prefiriendo fondos blandos de arenas. Estos datos concuerdan con los aportados por Vinuesa (2005a), quien asegura que los crustáceos decápodos son esencialmente vagantes y prefieren, en algunos casos, tipos de fondo diferentes a lo largo de su ciclo de vida. Dos ejemplos de este tipo de reparto, donde los juveniles habitan el sublitoral poco profundo y los adultos de mayor tamaño, profundidades mayores, son la falsa centolla $L$. confundens y el cangrejo araña E. latreillei. Otros permanecen en los mismos fondos durante toda su vida, como el caso de los cangrejos intermareales $P$. spinosulum y $H$. planatus. También observó esto en la especie cavadora $H$. (H.) polydactyla, de fondos arenosos o areno-fangosos, hecho que pudo ser reconocido en el estuario durante el estudio.

Con relación a la frecuencia observada para cada especie, puede decirse que los valores altos de $H$. planatus y $P$. spinosulum se deben principalmente a que se tratan de especies residentes que tienen poblaciones estables en la zona durante todo el año; mientras que los anomuros sólo concurren a las zonas intermareales en época reproductiva, y su captura será diferencial según el período reproductor en que se encuentren. Respecto a E. latreillei, su frecuencia fue baja dado que solo fueron muestreados individuos juveniles y sub-adultos, pero no adultos de aguas más profundas. $H$. (H.) polydactyla presentó una frecuencia baja, posiblemente por tratarse de una especie cavadora y componente de la infauna más que una especie vagante.

Se observa un gradiente en la diversidad, desde la boca al interior del estuario, viéndose una diferencia importante en cuanto al número de especies presentes en cada estación y a su abundancia en las mismas. La estación exterior (Punta Loyola-E4) fue donde se registraron todas las especies aunque en abundancias variables. Las estaciones medias (Río Gallegos-E2 y Río Chico-E3) presentaron únicamente dos especies que variaron significativamente su 
abundancia en cada estación. En cuanto a la estación interior (Güer Aike-E1) sólo presentó una especie. Los anomuros sólo estuvieron presentes en la estación exterior (Punta LoyolaE4) al igual que el estomatópodo. Puede verse que cada estación presentó fondos diversos, que afectaron directamente a la riqueza y abundancia de los crustáceos en las mismas, y por consiguiente su distribución dentro del estuario.

El hecho de que $H$. planatus presentara solo hembras en el área estudiada, es un hecho documentado por Vinuesa y Ferrari (2003) y Vinuesa (2005b) para la ría de Puerto Deseado (Santa Cruz, Argentina), y es un suceso que se repite en el estuario del río Gallegos. Para los anomuros P. granulosa y L. confundens, su migración reproductiva es diferencial en los sexos, apareciendo los machos seguido de las hembras como observaron Lovrich et al. (2000).

Se puede concluir indicando que la riqueza y abundancia de las especies de crustáceos decápodos y estomatópodos dentro del estuario del río Gallegos es baja en comparación a la que presentan diversos autores para el mar argentino, existiendo una variedad de hábitats y fondos que provocan un asentamiento diferencial de los crustáceos. Sin embargo, pueden llegar a encontrarse algunas especies aún no registradas de origen sur pacífico, como propone Torres (2006), que han presentado migraciones recientes.

\section{AGRADECIMIENTOS}

Los autores agradecen a Daniel Grima (Laboratorio de Cartografía, Teledetección y GIS, UNPA-UARG) por la realización de la cartografía, a Amanda Manero por facilitar el acceso a la Colección de Referencia de la Cátedra de Zoología (UARG-UNPA), a José R. Torres y Magdalena Campagnani por colaborar en el traslado a las zonas de muestreo, a Daniela F. Alegre Ilnao por su ayuda en la traducción, y a los dos revisores anónimos por las sugerencias al manuscrito.

\section{RESUMEN}

Estudiamos la diversidad y distribución de los crustáceos estomatópodos y decápodos en el estuario del río Gallegos, Argentina. En todas las estaciones se registró la presencia de decápodos, pero solo en la estación exterior se registraron estomatópodos y anomuros. La diversidad, fue marcadamente mayor en la estación exterior. Se registra un total de seis especies (cinco decápodos y un estomatópodo), indicando que la riqueza de crustáceos en el estuario es baja con relación a la señalada para el mar argentino. Su abundancia es variable en las estaciones, dependiendo del tipo de fondo.

Palabras clave: distribución, diversidad, estuario del río Gallegos, provincia de Santa Cruz, Argentina.

\section{REFERENCIAS}

Boschi, E.E. 1964. Los crustáceos decápodos Brachyura del litoral bonaerense. Bol. Inst. Biol. Mar. 6: 1-76.

Boschi, E.E. 1979. Geographic distribution of Argentinian marine decapod crustaceans. Instituto Nacional de Investigación y Desarrollo Pesquero (INIDEP). Contribución Científica 367. Bull. Biol. Soc. Wash. 3: 134-143.

Boschi, E.E. 1997. Crustáceos decápodos, p. 9-47. In M.B. Cousseau (ed.). Peces, Crustáceos y Moluscos Registrados en el Sector del Atlántico Sudoccidental Comprendido entre $34^{\circ}$ y $55^{\circ} \mathrm{S}$, con Indicaciones de las Especies de Interés Pesquero. Instituto Nacional de Investigación y Desarrollo Pesquero (INIDEP), Informe Técnico 5. Mar del Plata, Argentina.

Boschi, E.E. 2000. Species of decapod crustaceans and their distribution in the American marine zoogeographic provinces. Rev. Invest. Des. Pesq. 13: 7-136.

Boschi, E.E., C.E. Fischbach \& M.I. Iorio. 1992. Catálogo ilustrado de los crustáceos estomatópodos y decápodos marinos de Argentina. Frente Marit. 10: 7-94.

Braccalenti, J.C. 2000. Recursos pesqueros marinos de Santa Cruz, p. 332-346. In El Gran Libro de la Provincia de Santa Cruz. Milenio-ALFA Centro Literario, Patagonia, Argentina.

Caille, G.M. 1996. La pesca artesanal en las costas de Patagonia: hacia una visión global. Informes Técnicos del Plan de Manejo Integrado de la Zona Costera Patagónica-Fundación Patagonia Natural 7: 14.

Caille, G., S. Ferrari \& C. Albrieu. 1995. Los peces de la ría de Gallegos, Santa Cruz, Argentina. Natur. Patagónica 3: 191-194. 
Ferrari, S. 2000. La pesquería artesanal en la zona costera de Santa Cruz, p. 966-979. In El Gran Libro de la Provincia de Santa Cruz. Milenio-ALFA Centro Literario, Patagonia, Argentina.

Ferrari, S. \& C. Albrieu. 2000. Viajeros incansables: chorlos, playeros y otros caradriformes p. 360369. In El Gran Libro de la Provincia de Santa Cruz. Milenio-ALFA Centro Literario, Patagonia, Argentina.

Lovrich, G.A., M. Perroni, J.H. Vinuesa, F. Tapella \& A. Chizzini. 2000. Ocurrencia de la centolla Lithodes confundens (Decapoda) en el submareal de bahía Grande, Santa Cruz. IV Jornadas Nacionales de Ciencias del Mar. Puerto Madryn, Chubut, Argentina. p. 84.

Pellanda, L.A. \& P.F. Fernández. 2000. Los recursos pesqueros continentales, p. 932-964. In El Gran Libro de la Provincia de Santa Cruz. Milenio-ALFA Centro Literario, Patagonia, Argentina.

Perroni, M.O. 2000. La pesca costera manual en el estuario de Río Gallegos, p. 978. In El Gran Libro de la Provincia de Santa Cruz. Milenio-ALFA Centro Literario, Patagonia, Argentina.

Pittaluga, S. 2003. Alimentación de Eleginops maclovinus durante el otoño de 2002 en el estuario del río Gallegos (Santa Cruz). II Jornadas Patagónicas de Mallines y Humedales. UNPA-UARG, Argentina. p. 27.

Retamal, M.A. 1981. Catálogo ilustrado de los crustáceos decápodos de Chile. Gayana Zool. 44: 1-110.

Retamal, M.A. 2000. Decápodos de Chile (CD-ROM) ETI-Universidad de Concepción. Springer-Verlag, Berlin, Alemania.

Romero, O.Z. 2002. Guía de biodiversidad $\mathrm{N}^{\circ}$ 2. Vol. 1. Macrofauna y algas marinas. Crustáceos. Centro Regional de Estudios de Educación Ambiental (CREA). Universidad de Antofagasta, Antofagasta, Chile. 77 p.

Spivak, E.D. 1997. Los crustáceos decápodos del Atlántico sudoccidental $\left(25^{\circ}-55^{\circ} \mathrm{C}\right)$ : distribución y ciclos de vida. Invest. Mar. 25: 69-91.
Torres, E.R. 2005. Crustáceos estomatópodos y decápodos del estuario del río Gallegos, Santa Cruz- Argentina. XI Congreso Latinoamericano de Ciencias del Mar (XI COLACMAR) y XXI Congreso de Ciencias del Mar Chile, Viña del Mar, V Región, Chile. p. 376.

Torres, E.R. 2006. Primer registro de Pinnixa valdiviensis (Rathbun, 1907) (Decapoda: Pinnotheridae) en aguas costeras de Argentina. Invest. Mar. 34: 175-179.

Torres E.R. \& F.A. Vargas. 2005. Dieta del macá tobiano (Podiceps gallardoi) en el estuario del río Gallegos, Santa Cruz, Argentina. Rev. Nuestras Aves 50: 33-35.

Torres, E.R., A. Manero \& F.A. Vargas. 2005. Relevamiento de la biodiversidad costera del estuario del río Gallegos (Santa Cruz, Argentina): invertebrados bentónicos. XI Congreso Latinoamericano de Ciencias del Mar (XI COLACMAR) y XXI Congreso de Ciencias del Mar Chile. Viña del Mar, V Región, Chile. p. 377.

Torres, E.R., A. Manero \& F.A. Vargas. 2006. Listado sistemático y distribución temporal de los peces del estuario del río Gallegos (Santa Cruz, Argentina). An. Inst. Patagonia (Chile). 34: 59-64.

Vinuesa, J.H. 1977. Aportes al conocimiento de los crustáceos decápodos de Tierra del Fuego con algunas observaciones zoogeográficas. Physis Sec. A. 36 (92): 9-19.

Vinuesa, J.H. 2005a. Distribución de crustáceos decápodos y estomatópodos del golfo San Jorge, Argentina. Rev. Biol. Mar. Oceanog. 40: 7-21.

Vinuesa, J.H. 2005b. El ciclo de vida de las hembras del cangrejo Halicarcinus planatus (Decapoda, Hymenosomatidae) en Puerto Deseado (Santa Cruz, Argentina). XI Congreso Latinoamericano de Ciencias del Mar (XI COLACMAR) y XXI Congreso de Ciencias del Mar Chile, Viña del Mar, V Región, Chile. p. 101.

Vinuesa, J.H. \& L. Ferrari. 2003. Reproducción del cangrejo Halicarcinus planatus en la ría de Puerto Deseado. V Jornadas Nacionales de Ciencias del Mar. Mar del Plata, Buenos Aires, Argentina. p. 184. 
